\title{
Instrumentierung und Fusion nach zervikalem Trauma
}

\author{
Stefan Matschke, Martin Adams, Andreas Wentzensen
}

\section{Zusammenfassung}

In Abhängigkeit der Lokalisation einer Verletzung im oberen oder unteren HWS-Bereich werden unterschiedliche Zugangswege und Instrumentationssysteme zur Stabilisation verwendet. Hierbei haben sich für den oberen $\mathrm{Ab}$ schnitt der HWS von $\mathrm{CO}-\mathrm{C} 3$ folgende Verfahren durchgesetzt: Bei Instabilitäten im okzipitozervikalen Übergang sowie bei atlantoaxialen Instabilitäten kommen ausschließlich posteriore $\mathrm{Fu}-$ sionsoperationen mit Plattenstabsystemen, Cerclage-, Hakensystemen oder Schraubensystemen zur Anwendung. Die Fusion wird über autologe oder homologe Knochentransplantate erreicht. Ausnahmen stellen instabile Densfrakturen vom Typ Anderson II dar. Hier erfolgt die Frakturversorgung mit einer kanülierten Zugschraubenosteosynthese über einen ventralen Zugang. Traumatische Wirbelsäulenverletzungen, die im mittleren und unteren Abschnitt der HWS lokalisiert sind (C4 Th1/Th2), stellen eine Indikation für eine ventrale Fusionsoperation dar. Zur Sicherung der Fusion, die in der Regel mit einem Beckenkammblock erfolgt, ist eine Instrumentierung mit einer Plattenosteosynthese erforderlich. In seltenen Fällen bei verbleibender posteriorer Instabilität ist eine kombinierte Operationstechnik mit einem additiven dorsalen Schraubenstabsystem erforderlich. Für alle operativen Therapiemaßnahmen gilt das Ziel, nach erfolgter anatomischer Reposition und Dekompression eine sichere und repositionsverlustfreie Ausheilung und Fusion zu erreichen.

\section{Stabilisation and Spinal Fusion for Cervical Injuries}

Depending on the localisation of an injury in the upper or lower cervical spine region, different approaches and instrumentation systems are used for stabilisation. The following procedures are now established for the upper segment of the cervical spine from $\mathrm{CO}-\mathrm{C} 3$ : For instabilities of the occipito-cervical transition as well as for atlanto-axial instabilities posterior fusion operations with plate and rod systems, wiring, hook systems or screw systems are used exclusively. Fusion is achieved with the help of autologous or homologous bone grafts. Unstable Anderson type II fractures of the dens are an exception. In such cases the fracture is managed by means of a cannulated tension screw osteosynthesis system via a ventral access. Traumatic spinal injuries located in the middle and lower segments of the cervical spine (C4Th1/Th2) represent an indication for a ventral fusion operation. The fusion which is usually achieved with an iliac crest graft requires instrumental stabilisation with a plate osteosynthesis. Only in the rare cases of a remaining posterior instability is a combined operative technique with an additive dorsal screw/rod system necessary. For all surgical therapeutic interventions the aim is to achieve an anatomic repositioning and a secure healing and fusion without loss of position.

\section{Einleitung}

Bei der Versorgung instabiler Halswirbelverletzungen werden der operative Zugangsweg und die operationstechnischen Möglichkeiten bestimmt durch das Verletzungsausmaß, die resultierende Instabilität sowie die Verletzungshöhe.

So erfolgt für den oberen Abschnitt der HWS (CO-C3) anatomisch bedingt eine angestrebte Fusion und Stabilisierung über isolierte Schrauben oder in Kombination mit Drahtsystemen. Mit Einfüh-

OP-JOURNAL 2009; 25: 20-26

(c) Georg Thieme Verlag KG Stuttgart · New York DOI 10.1055/s-0029-1185496 rung der transartikulären Verschraubung C2/C1 nach Magerl und Seemann 1986 [16] konnte eine deutlich höhere Stabilität gegenüber den bis dahin isoliert durchgeführten Cerclagetechniken nach Brooks [4] bzw. Gallie [11] erreicht werden.

Alle Verletzungen im Bereich der oberen HWS mit Ausnahme der Densfrakturen vom Extensionstyp werden über dorsale Fusionsoperationen stabilisiert. Bei Erfordernis der Fusion okzipitozervikal wird diese unter Verwendung eines dorsalen Plattenschraubensystems in Kombination mit einer Knochenanlagerung erreicht.
Halswirbelsäulenverletzungen, die den mittleren und unteren Abschnitt betreffen, können, als häufigste operative Therapiemaßnahme, durch eine alleinige ventrale Fusion über Plattensysteme, seltener ergänzt durch ein dorsales Schraubenstabsystem, kurz oder lang streckig operativ stabilisiert werden. Die angestrebte Fusion wird durch einen Knochenblock erreicht. Zur Verringerung der Morbidität nach Beckenkammspanentnahme kommen bei monosegmentalen Fusionen auch Cages aus verschiedenen Materialien und Designs zur Anwendung [17].

Winkelstabile Platten erleichtern die operative Technik, eine höhere Stabilität gegenüber nicht winkelstabilen Implan- 


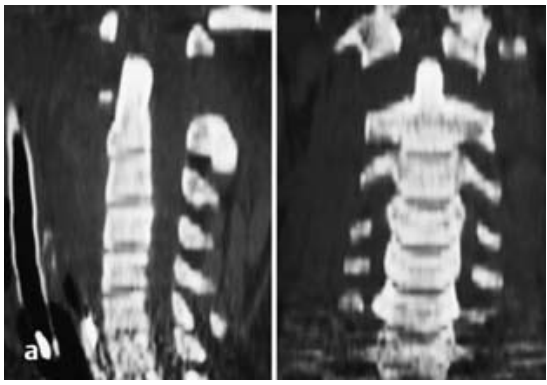

Abb. 1 a Atlantoaxiale Dissoziation (AAD).

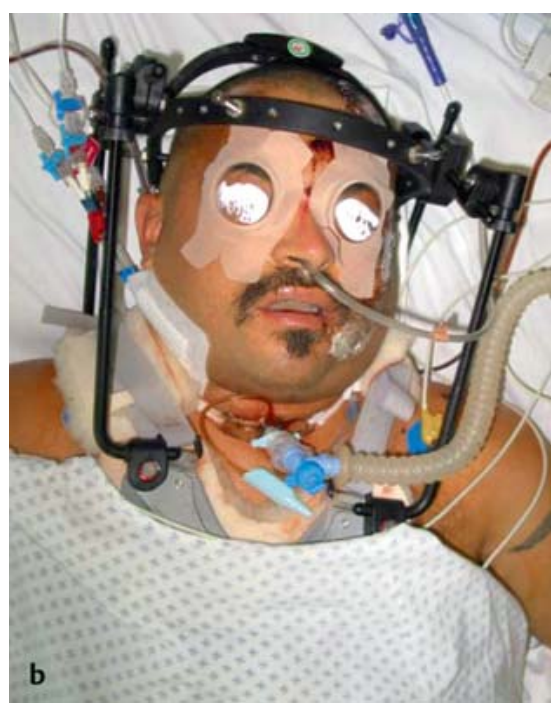

Abb. 1 b Geschlossene Reposition und Anlage eines Halo-Fixateurs.

taten mit bikortikaler Schraubenlage ergibt sich nicht.

Hochinstabile Verletzungen erfordern ein kombiniertes ventrodorsales oder dorsoventrales Vorgehen. Für die dorsale Instrumentierung haben sich verschiedene Schraubenstabsysteme etabliert. Als Verankerungsmöglichkeit der dorsal zuggurtenden Implantate wird die Massa lateralis verwendet. Einen größeren Halt bietet eine transpedikuläre Schraubenlage bei entsprechender Pedikelgröße. Für eine transpedikuläre Schraubenpositionierung ist eine intraoperative Navigation oder 3-dimensionale Bilddarstellung erforderlich. Hakensysteme können alternativ zu Massa-lateralis-Schrauben oder zur Instrumentierung auf den Bogen C7 für eine posteriore Instrumentierung zur Anwendung kommen.

Bei instabilen Kombinationsverletzungen der unteren HWS und der oberen BWS, die über Th1/2 hinausreicht, erfolgt eine zervikothorakale Instrumentierung von dorsal mit einem Schraubenstabsystem.
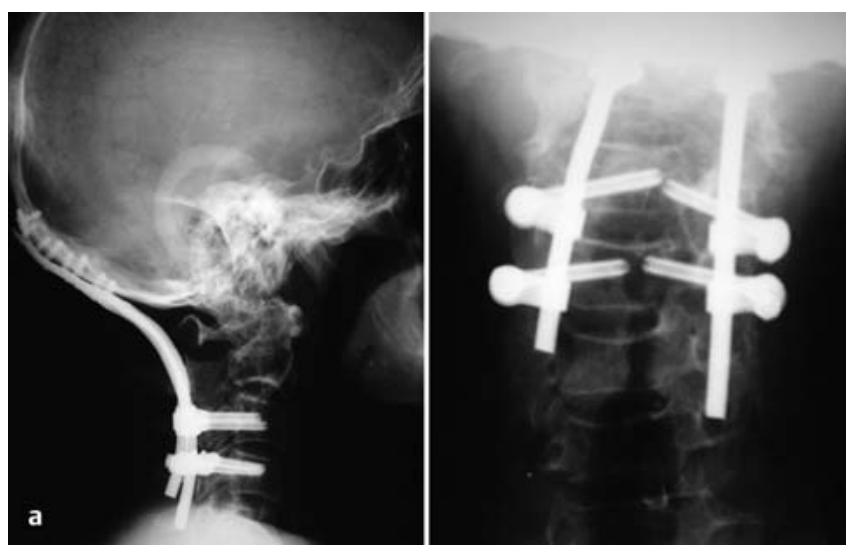

Abb. 2a Ausheilungszustand nach okzipitozervikaler Fusion, transpedikuläre Schraubenbesetzung $\mathrm{C} 3$ und $\mathrm{C} 4$

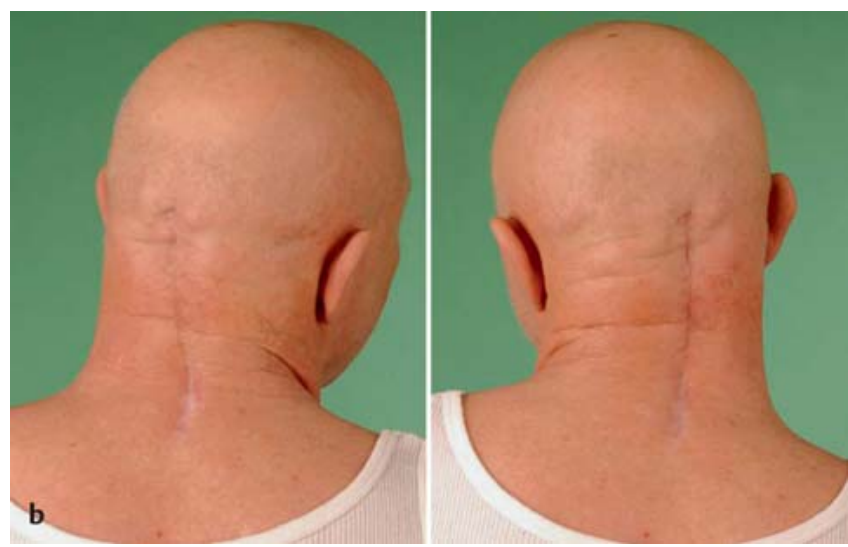

Abb. 2b Funktionsaufnahmen Kopfdrehung rechts/links nach okzipitozervikaler Fusion.

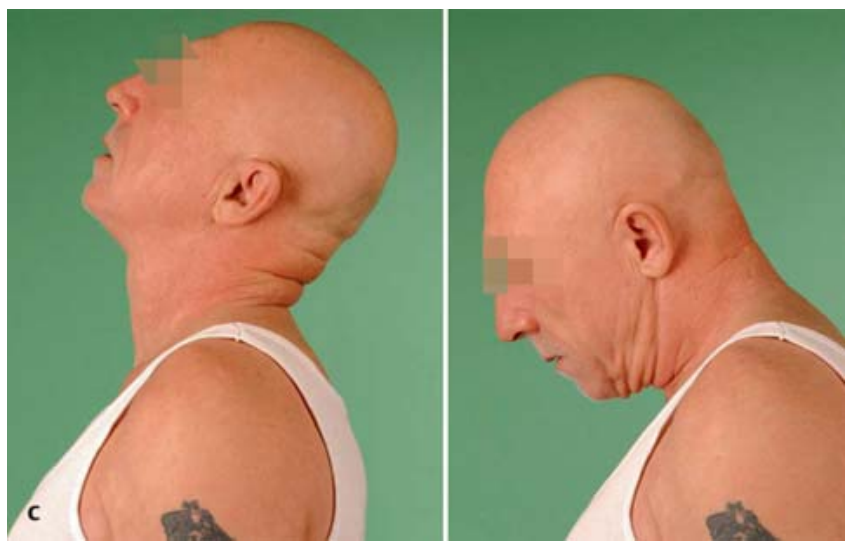

Abb. 2c Funktionsaufnahmen Re- und Inklination nach okzipitozervikaler Fusion.

\section{Dorsale zervikale Instrumentierung im Bereich der oberen HWS}

Verletzungen und Klassifikationen der oberen HWS (CO-C3)

1. atlantookzipitale Dissoziation (AOD)

2. atlantoaxiale Dissoziation (AAD)

3. Atlasfrakturen

4. Densfrakturen

Die atlantookzipitale Dissoziation (AOD) stellt wie die atlantoaxiale Dissoziation $(A A D)$ eine sehr seltene und schwere Verletzung dar mit hoher Letalitätsrate. Durch ein gut funktionierendes Ret- tungssystem erreichen heute häufiger Patienten mit einer AOD bzw. AAD lebend die Klinik. Begleitend für diese Verletzungen, gleichzeitig auch für die Prognose und Therapie entscheidend, sind zusätzlich vorhandene Schädel-HirnVerletzungen. Durch Zerreißungen und Dissektionen der Aa. vertebralis kommt es zu starken Einblutungen in die Halsweichteile, Hirninfarkte können als Zeichen der Mangeldurchblutung resultieren. Für diese hochinstabilen Patienten kommt in der Initialphase nur die temporäre Stabilisierung nach Reposition der anatomischen Achse über einen Halo-Fixateur zur Anwendung (Abb. 1 b). 


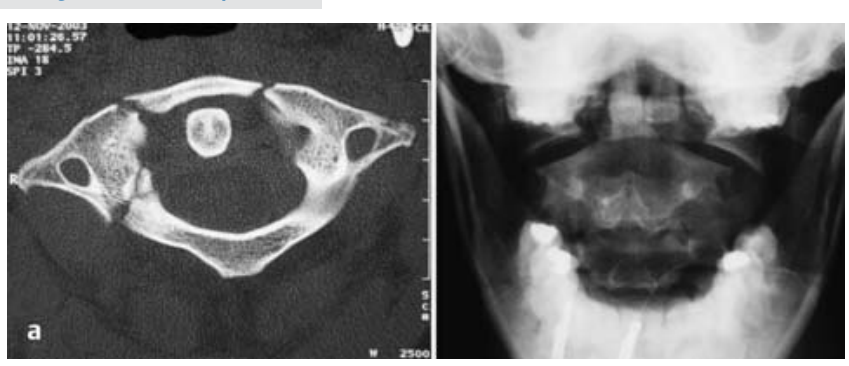

Abb. 3 a JeffersonFraktur, vordere und hintere Atlasbogenfraktur mit Auseinanderweichen der Massa lateralis (instabil).

Nach Stabilisierung der Vitalparameter des Patienten erfolgt im weiteren kurzfristigen Verlauf nach wenigen Tagen die definitive Stabilisierung und Instrumentierung der Verletzung.

Aufgrund der anatomischen Gegebenheiten wird bei der atlantookzipitalen Dissoziation über ein dorsales PlattenSchraubensystem eine okzipitozervikale Fusion angestrebt (Abb. 2 a bis c).

Wenn immer möglich, erfolgt die Fixierung zervikal durch eine transartikuläre Verschraubung C2/C1 nach Magerl [2, $12,16]$. Anzustreben ist eine möglichst kurz streckige Fusion über wenige Bewegungssegmente. Mit Ausnahme bei Kindern ist die Anlagerung von Spongiosa zwischen dem Okziput und dem Atlasring bzw. Axisring erforderlich, um eine Fusion zu erreichen.

Bei Luxation und Zerreißung des Bewegungssegments $\mathrm{C} 1 / \mathrm{C} 2$ im Sinne der atlantoaxialen Dissoziation (Abb.1a) erfolgt die definitive operative Stabilisierung nach Reposition von dorsal durch eine transartikuläre Verschraubung der Gelenke C2/C1 nach Magerl in Kombination mit Fixierung der Wirbelbögen von Atlas und Axis über eine Drahtcerclage. Auch hier ist zur definitiven Fusion ein Knochenblock erforderlich, der nach Anfrischung der Wirbelbögen $\mathrm{C} 1$ und $\mathrm{C} 2$ zwischen diesen positioniert und durch die Cerclage fixiert wird. Hierbei unterscheidet man zwischen 2 verschiedenen Techniken.

Bei der OP-Methode nach Brooks [4] werden 2 Cerclagen zwischen den Wirbelbögen angebracht und fixieren rechts und links der Mittellinie jeweils einen kortikospongiösen Span (Abb.5b). Bei der OP-Methode nach Gallie [11] fixiert eine Cerclage einen kortikospongiösen Span, über den die Fusion angestrebt wird.

Biomechanisch bietet die OP-Methode nach Brooks eine höhere Stabilität.
Bei den Atlasfrakturen werden 5 verschiedene Frakturtypen unterschieden [10]. Hiervon ist nur eine Frakturform instabil und bedarf der operativen Stabilisierung.

\section{Typ 1}

Isolierte Fraktur des vorderen Atlasbogens (seltene Abrissverletzung durch Zug des M. longus colli im Rahmen von Hyperextensionsmechanismen). Diese Verletzung ist als stabil einzustufen und bedarf keiner operativen Versorgung.

\section{Typ 2}

Isolierte Fraktur des hinteren Atlasbogens (immer doppelseitig). Auch dieser Verletzungstyp bedarf keiner operativen Stabilisierung, da ausreichende Stabilität gewährleistet ist. $\mathrm{Zu}$ achten ist auf weitere Verletzungen der HWS, die häufig mit vorhanden sind.

\section{Typ 3}

Entspricht der kombinierten Form aus Frakturen des vorderen und hinteren Atlasbogens. Sie trägt den Eigennahmen Jefferson-Fraktur [14] nach ihrem Erstbeschreiber.

Instabil und damit operationspflichtig wird dieser Verletzungstyp dann, wenn es zum Auseinanderweichen der Massae laterales kommt. (Abb. 3a)

Typ 4

Selten, isolierte Fraktur Massae laterales - stabil.

\section{Typ 5}

Selten, isolierte Fraktur Processus transversus - stabil.

Die empfohlene Therapie bei stabilen Atlasfrakturen ist die temporäre äußere Ruhigstellung in einer harten Zervikalstütze für 4 Wochen.

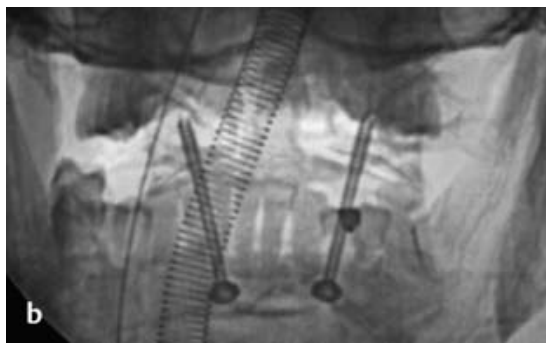

Abb. 3b Jefferson-Fraktur, transartikuläre Verschraubung nach Magerl.

Liegen dislozierte Massae laterales atlantis vor, als Kennzeichen der instabilen Fraktur, besteht nach gegenwärtiger Lehrmeinung die Empfehlung zur alleinigen transartikulären Verschraubung C2/C1 nach Magerl [12,16] (Abb.3b) ohne zusätzliche Fusion. In Einzelfällen kann auch die Ausheilung nach Anlage eines Halofixateurs erreicht werden.

\section{Densfrakturen}

Die Densfraktur ist eine typische Wirbelsäulenverletzung des alten Menschen.

Bei den über 70-Jährigen rangiert die isolierte Densfraktur an erster Stelle aller HWS-Verletzungen.

In der Einteilung nach Anderson und D'Alonso [7] stellt die Verletzung vom Typ II (Fraktur des Processus odontoideus oberhalb seiner Basis) aufgrund der hohen Pseudarthroserate nach konservativer Therapie eine Indikation zur operativen Therapie dar.

Zu unterscheiden sind hierbei die häufigeren Extensionsfrakturen (73\%) (Aufprall mit der Stirn) von den Flexionsfrakturen (19\%) (Aufprall auf den Hinterkopf).

Während die Extensionsfrakturen nach geschlossener Reposition von ventral mit einer Zugschraubenosteosynthese in kanülierter Technik versorgt werden (siehe unten), können Flexionsfrakturen oder Frakturen mit einer ausgedehnten Trümmerzone nicht in dieser Technik stabilisiert werden. Hier ist bei hochgradiger Instabilität ein dorsales Vorgehen indiziert [8]. Nach geschlossener Reposition erfolgt die Stabilisierung über eine transartikuläre Verschraubung $\mathrm{C} 2 / \mathrm{C} 1$ nach Magerl $[12,16]$ in Kombination mit der OP nach Gallie [11] oder Brooks [4].

Eine seltene hochinstabile Form mit kombinierter segmentaler Instabilität stellt die transdentale Luxationsfraktur 

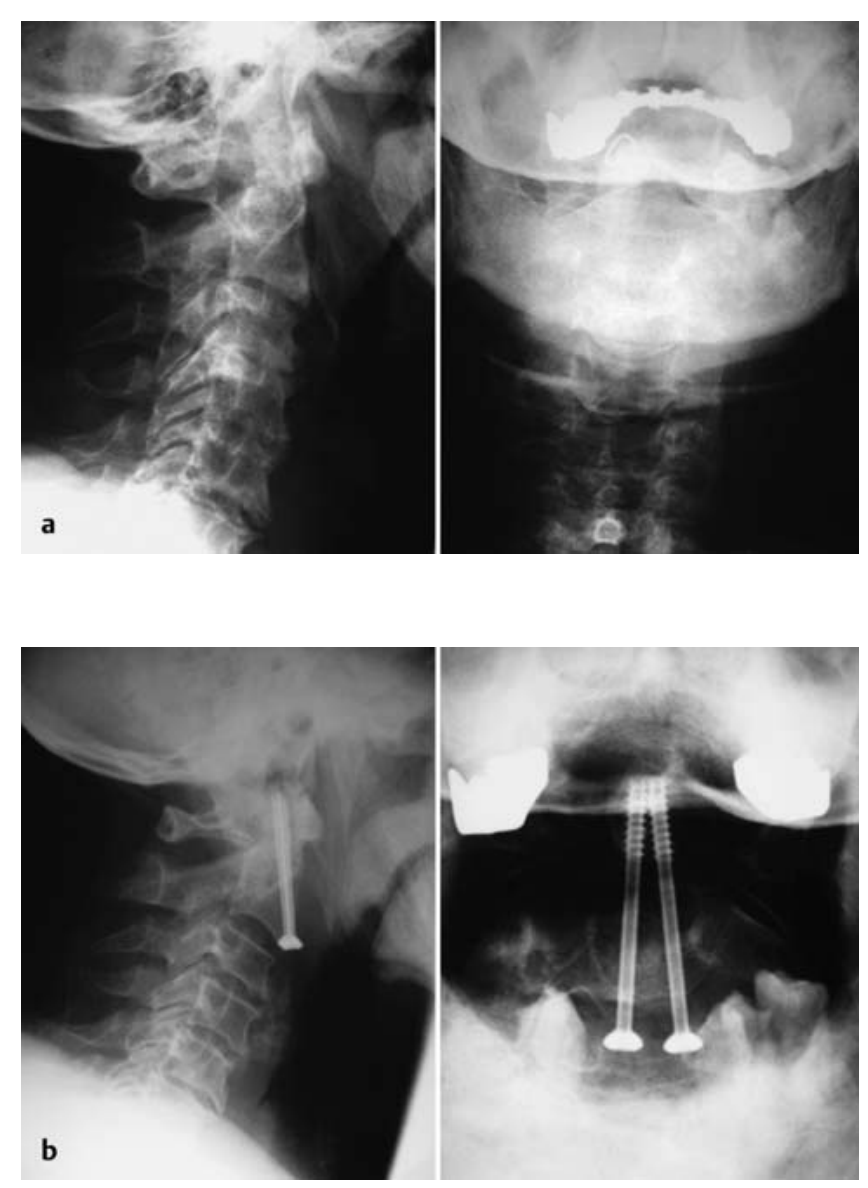

Abb. 4b Operative Stabilisierung nach geschlossener Reposition durch kanülierte Schraubenosteosynthese.

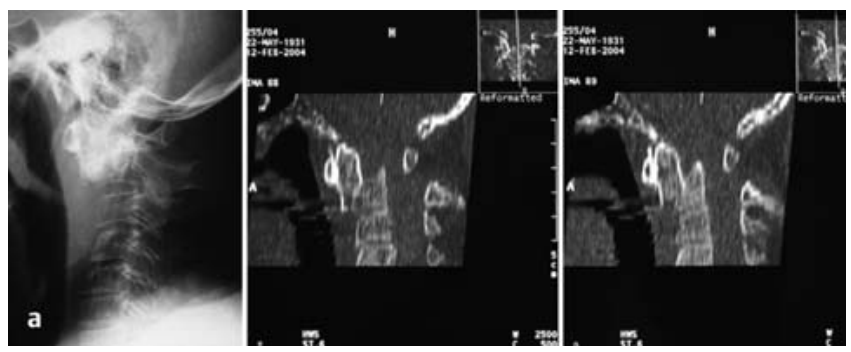

Abb. 5a Transdentale Luxationsfraktur.
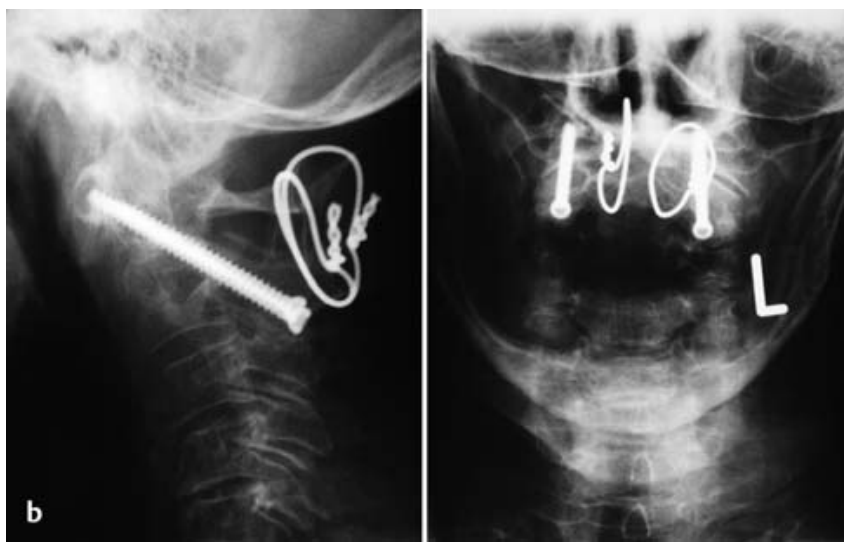

Abb.5b Operative Versorgungsbilder mit dorsaler transartikulärer Verschraubung nach Magerl $\mathrm{C} 2 / \mathrm{C} 1$ und Fusionsoperation nach Brooks. dar (Abb. 5a). Sie ist gekennzeichnet neben der Fraktur und Luxation des Dens im Sinne einer Flexionsfraktur durch die vorliegende Zerreißung und dorsale Instabilität, die sich radiologisch durch einen vergrößerten Abstand der dorsalen Bögen zwischen Atlas und Axis zeigt. Die erforderliche operative Stabilisierung gelingt bei dieser hochinstabilen Verletzung ausschließlich von dorsal durch eine transartikuläre Verschraubung C2/C1 nach Magerl $[12,16]$ in Kombination mit einer OP nach Gallie [11] oder Brooks [4] (Abb. 5b).

Neuere Instrumentationssysteme erlauben bei atlantoaxialer Instabilität, die transartikuläre Verschraubung C2/C1 nach Magerl mit einer Atlasklammer als Cerclageersatz zu koppeln (Abb. 6).

Ist eine transartikuläre Verschraubung C2/C1 nach Magerl aufgrund anatomischer Varianten nicht sicher durchführbar, stellt die C1/C2-Verschraubung nach Harms [13] eine Alternative dar. Die Stabilisierung erfolgt hierbei über ein Multiaxialschraubensystem und Stäbe und kann nach Erfordernis mit einer Knochenanlagerung kombiniert werden (Abb. 7).

\section{Ventrale zervikale Instrumentierung im Bereich der oberen HWS}

\section{Densfrakturen}

Die gebräuchlichste Einteilung der Densfrakturen erfolgt nach Anderson und D’Alonso [7] mit 3 Verletzungstypen.

Eine orientierende Therapieempfehlung lässt sich anhand der Typeinteilung geben.

Hierbei entspricht die seltene Typ-I-Fraktur einer knöchernen Ausrissverletzung der Ligg. alaria, auch als Densspitzenfraktur bezeichnet. Sie tritt eher im Rahmen einer atlantookzipitalen Dissoziation auf. Die einzuschlagende Therapie ist in Abhängigkeit von der Grundverletzung auszuwählen, da es sich im strengen Sinne nicht um eine Densfraktur handelt.

Die am häufigsten beim alten Menschen zu findende Typ-II-Fraktur ist eine potenzielle instabile Fraktur mit einer hohen Affinität zur Entstehung einer Pseudarthrose. Sie lokalisiert sich auf den Processus odontoideus oberhalb seiner Basis. Unverschobene Querfrakturen bergen ein Potenzial der Instabilität. Durch- 
leuchtungsuntersuchungen unter Extensions-Flexionsbewegungen geben hier Auskunft über den Grad der Stabilität und ermöglichen die Entscheidung, ob eine konservative Therapie mit einer Zervikalstütze für 6 Wochen eingeleitet werden kann oder die ventrale Schraubenosteosynthese erforderlich ist.

Die heute gebräuchlichste ventrale Zugschraubenosteosynthese in kanülierter Technik (Abb.4a und b) ist geeignet zur Stabilisierung der Extensionsfrakturen, da nach Reposition der Frakturspalt durch die Schrauben geschlossen werden kann.

Typ-III-Frakturen lokalisieren sich auf die Densbasis im spongiösen Bereich und besitzen ein ausreichendes Potenzial zur knöchernen Heilung unter konservativen Therapiemaßnahmen.

\section{Traumatische Spondylolisthese des Axis}

Pathomorphologisch handelt es sich bei diesen Verletzungen um eine Kombination aus Fraktur des Axisrings und einer Subluxation im Segment C2/C3.

Erstmals wurde diese Verletzung bei Delinquenten, die durch Strangulation hingerichtet wurden, beschrieben, weshalb sich bis heute der Begriff hanged man fracture als Eigenname erhalten hat.

Bei der Verletzungsentstehung sind jedoch axiale Kompressionskräfte in Kombination mit rotatorischen Komponenten maßgebend, sodass heute besser der Begriff der traumatischen Spondyloliszitis des Axis verwendet werden sollte.

Eine der gebräuchlichsten Einteilungen erfolgt nach Effendi [9], wobei 3 Verletzungstypen unterschieden werden.

Bei der Verletzung vom Typ 1 findet sich charakteristisch keine oder nur eine geringe Subluxation im Bandscheibensegment $\mathrm{C} 2 / \mathrm{C} 3$, wobei die Fraktur des Axisrings jeden Teil betreffen kann. Sie stellt den häufigsten Frakturtyp dar, ist stabil und kann der konservativen Therapie zugeführt werden.

Kennzeichnend für den Typ 2 sind die Verletzung und die Instabilität im Bandscheibensegment $\mathrm{C} 2 / \mathrm{C} 3$ in Kombination mit einer Fraktur des Axisrings. Effendi [9] unterscheidet in Abhängigkeit der Stellung des vorderen Wirbelkörperanteils 3 weitere Untertypen.
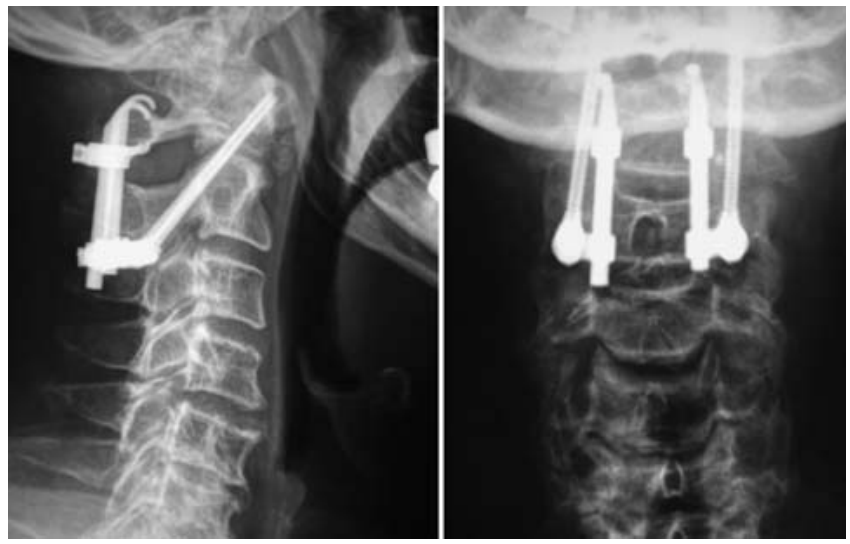

Abb. 6 Posteriore Fusion C1/C2 mit transartikulärer Verschraubung nach Magerl und Atlasklammer.
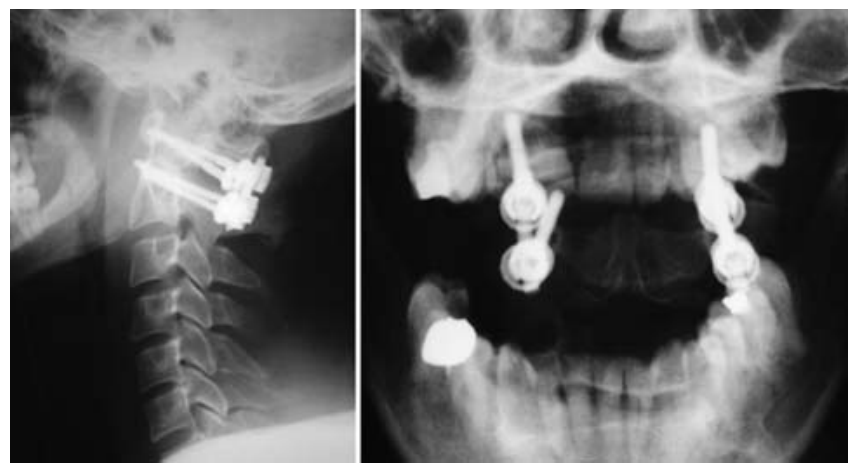

Abb. 7 Posteriore C1/C2-Verschraubung nach Harms.

Gering dislozierte Frakturen mit einer maximalen Kyphosierung bis zu $10^{\circ}$ und einer sagittalen Verschiebung $<5 \mathrm{~mm}$ können in der Regel konservativ behandelt werden. In seltenen Fällen kann die Stabilisierung über eine dorsale Verschraubung des fakturierten Axisrings erfolgen. Höhergradige Instabilitäten erfordern die Fusion des verletzten Bewegungssegments C2/C3. Nach geschlossener Reposition sind die Entfernung der verletzten Bandscheibe und eine ventrale Fusion mittels eines Beckenkammblocks und einer additiven Platteninstrumentierung erforderlich. Der operative Zugangsweg muss hierbei genau geplant werden, da in Abhängigkeit der anatomischen Gegebenheit bei muskelkräftigen Männern oder Patienten mit kurzem kräftigen Hals das Segment C2/C3 besser über eine Inzision etwa $1,5 \mathrm{~cm}$ entfernt vom Unterrand des Unterkiefers erreicht wird als über einen Standardzugang im Verlauf des Vorderrands des M. sternocleidomastoideus.

Beim Verletzungstyp 3, der instabilsten Form, liegt zusätzlich zu den Verletzungszeichen der Typ-2-Verletzung als Zeichen der einwirkenden Rotationskraft eine Verhakung der kleinen Wirbelgelenke ein- oder beidseitig vor.
Die operative Stabilisierung dieser hochinstabilen Verletzung erfolgt nach geschlossener oder offener Reposition durch eine interkorporelle ventrale Spondylodese C2/C3 analog dem Verletzungstyp 2.

\section{Ventrale zervikale Instrumentierung und Fusion bei Verletzungen der unteren HWS C4-C7/Th1}

Die isolierte Verwendung von Knochenspänen zur ventralen Fusion bei degenerativen Wirbelsäulenerkrankungen wurde 1958 von Cloward beschrieben [6]. Erst durch die Kombination mit einer ventralen additiven Plattenosteosynthese ist eine sichere Fusion traumatischer Frakturen zu erreichen $[1,3,5]$.

Die Vorteile des ventralen Zugangs sind in der schonenden anatomischen Zugangstechnik am Vorderrand des M. sternocleidomastoideus zu sehen (keine Durchtrennung oder Ablösung von Muskulatur im Vergleich zum dorsalen Zugang erforderlich) sowie der einfachen Möglichkeit der Darstellung und Dekompression des Spinalkanals von ventral.

Eine Begrenzung für ein ventrales Vorgehen können in seltenen Fällen verhakte Luxationen darstellen, die sich nicht von ventral reponieren lassen. Der ven- 

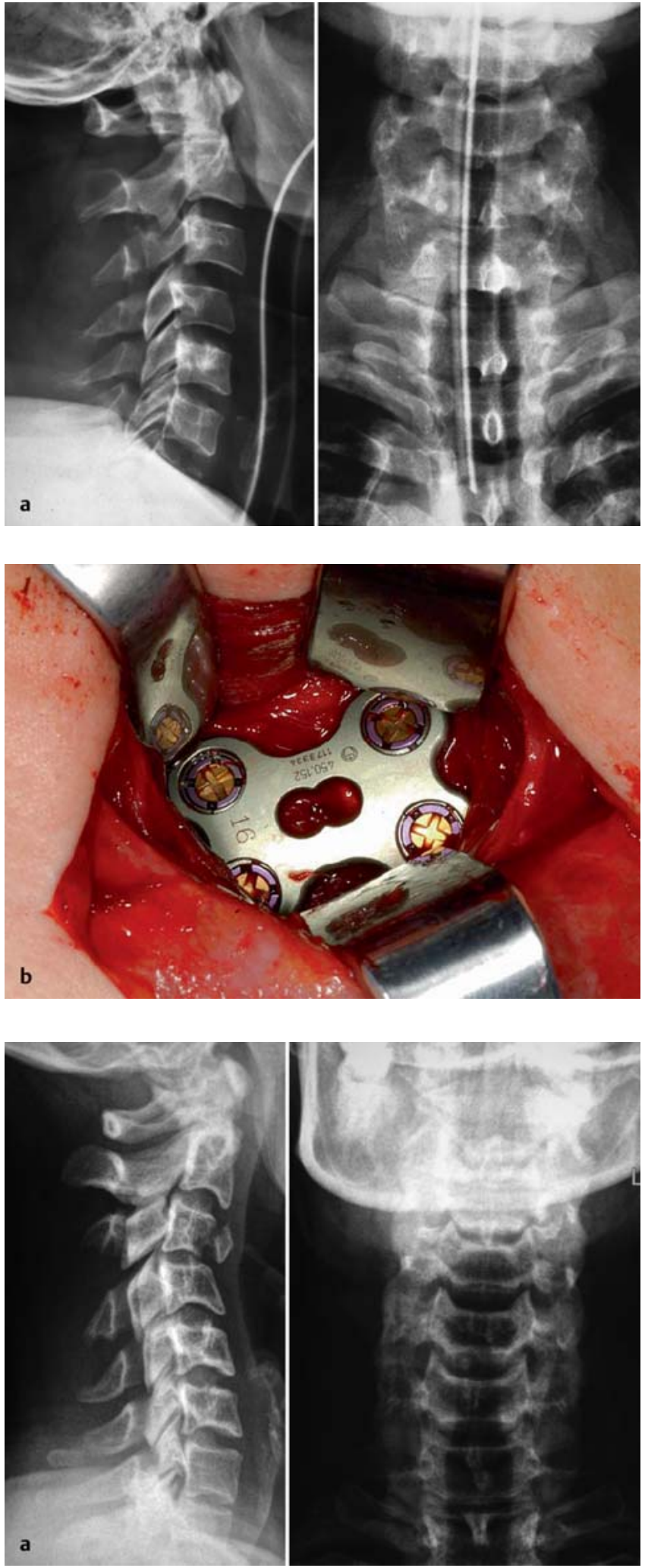

trale Zugang für Verletzungen des zervikothorakalen Übergangsbereichs ist bei schlanken Patienten bis auf Th1/Th2 möglich, unterhalb dieser Höhe ist eine Fixierung einer Platte nur über eine Sternotomie zu erreichen. Deshalb wird in
Abb. 9a FlexionsDistraktionsverletzung C3/C4 Typ B1.2 mit Typ-A-Fraktur.

Abb. 8a FlexionsDistraktionsverletzung ligamentär C3/ C4 mit Zerreißung des Diskus Typ B1.1.

Abb. 8b Intraoperatives Bild nach ventral monosegmentaler Fusion mit winkelstabiler Platteninstrumentierung.$$
\text { . }
$$

diesen Fällen günstigerweise eine dorsale zervikothorakale Instrumentierung angestrebt.

Entsprechend der heute gebräuchlichsten Frakturklassifikation im Bereich der mittleren und unteren HWS werden die Verletzungen in Typ-A-Kompressionsverletzungen, Typ-B-Distraktionsverletzungen und Typ-C-Rotationsverletzungen unterteilt [15].

Im Gegensatz zum thorakolumbalen Übergangsbereich, hier finden sich am häufigsten Kompressionsverletzungen (Typ A), gefolgt von Rotationsverletzungen (Typ C), betreffen die Verletzungen der mittleren und unteren HWS in den meisten Fällen eine Kombination aus vorderer und hinterer Säule im Sinne von Distraktionsverletzungen und Rotationsverletzungen (Abb.8a und b, 9a und b).

Einigkeit besteht heute darüber, dass eine zervikale Fusion bei allen instabilen Verletzungen angestrebt wird. Das betrifft alle Kompressionsverletzungen vom Typ A3 (Gruppe der Berstungsbrüche) sowie alle Distraktionsverletzungen und Rotationsverletzungen.

Für eine sichere zervikale Fusion ist die alleinige ventrale Spondylodese mit einer winkelstabilen oder nicht winkelstabilen Platte und einem kortikospongiösen Span ausreichend.

Die Verwendung von winkelstabilen Platten bietet den Vorteil, dass nur eine Kortikalis besetzt werden muss, um eine ausreichende Stabilität zu erreichen. Bei nicht winkelstabilen Platten muss im Wirbelkörper bikortikal gebohrt und die Schraube ebenso besetzt werden. Biomechanisch ergibt sich im Rahmen der Frakturheilung bei korrekter Verwendung beider Platten in der Frakturheilung kein Unterschied.

Keines der beiden Plattensysteme bietet jedoch ausreichenden Schutz und Sicherheit für die Einheilung des Spanes bei verbleibender dorsaler Instabilität (Abb. 10 a und b).

Wie das Beispiel der Flexionsdistraktionsverletzung Typ B2.3 in Höhe C5/C6 mit ausgeprägter ossärer und ligamentärer dorsaler Instabilität zeigt, ist die dorsale Instrumentierung über ein Schraubenstabsystem erforderlich.

Zwei Möglichkeiten der Schraubenverankerung von dorsal ergeben sich. Erstens die technisch leichtere Fixierung der Schrauben im Bereich der Massae laterales. Bei schlechter Knochenqualität kann es unter Umständen zum Ausreißen der Schrauben bei der Montage 

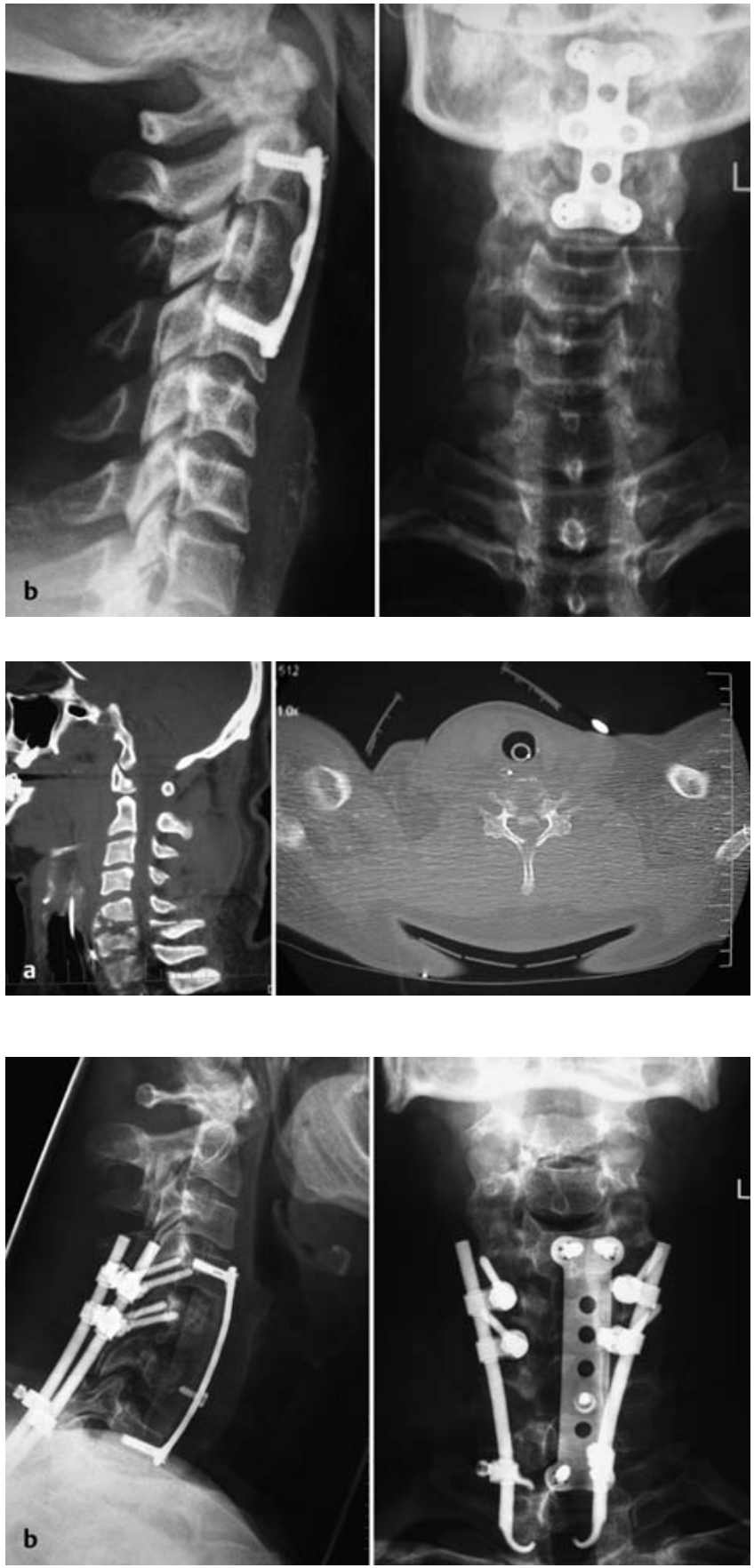

Abb. 10b Ventrodorsale Spondylodese C4-C7, ventral Korporektomie C5, C6 mit Dekompression durch Hinterkantenresektion, trisegmentale Fusion mit Beckenkammblock und winkelstabiler Platteninstrumentierung, 2-zeitig additive dorsale mehrsegmentale Instrumentierung.

kommen, da die Schraubengewindelänge in Abhängigkeit der Breite der Massae laterales nicht mehr als $12-16 \mathrm{~mm}$ beträgt. Mehr Sicherheit bieten in diesen Fällen mehrsegmentale dorsale Instrumentierungen. Ist eine größtmögliche Sicherheit für den Schraubenhalt bei der dorsalen Schraubenfixierung erforderlich, so bietet sich als zweite Möglichkeit eine transpedikuläre Schraubenverankerung an. Meist sind hierdurch kurz streckige, bisegmentale dorsale Instrumentierungen ausreichend und möglich. Die transpedikuläre Schraubenbesetzung im Bereich der Halswirbelsäule ist jedoch technisch anspruchsvoll aufgrund der unmittelbaren Lagebeziehung der Schrauben zwischen A. vertebralis und dem Myelon.

Die Möglichkeit der Navigation oder einer intraoperativen dreidimensionalen Darstellungsmöglichkeit mittels IsoC 3D oder Orbic sollte gegeben sein.

\section{Literatur}

${ }^{1}$ Aebi M, Zuber K, Marchesi D. Treatment of cervical spine injuries with anterior plating. Indications, techniques and results. Spine 1991; 16: 38-45
${ }^{2}$ Blauth M, Richter M, Lange U. Transarticular screw fixation of $\mathrm{C} 1 / \mathrm{C} 2$ in atlanto-axial instability. Comparison between percutaneous and open procedures. Orthopäde 1999; 28: 651-661

${ }^{3}$ Böhler J, Gaudernack T. Anterior plate stabilization for fracture dislocations of the lower cervical spine. J Trauma 1980; 20: 203-205

${ }^{4}$ Brooks AL, Jenkins EB. Atlantoaxial arthrodesis by the wedge compression method. J Bone Joint Surg [Am] 1978; 60: 279-284

${ }^{5}$ Caspar W, Pitzen T. Anterior cervical fusion and trapezoidal plate stabilization for re-do surgery. Surg Neuro 1999; 52: 345-352

${ }^{6}$ Cloward RB. The anterior approach for removal of ruptured discs. J Neurosurg 1958; 15: 602-617

7 Daum W, Archer CR. Fracture of the odontoid associated with pedicle fracture of the axis: a previously undescribed entity. J Trauma 1977; 17: 381-386

8 Dickman CA, Foley KT, Sonntag VK et al. Cannulated screws for odontoid screw fixation and atlantoaxial screw transarticular screw fixation. Technical note. J Neurosurg 1995; 83: 1095-1100

${ }^{9}$ Effendi B, Roy D, Cornish B et al. Fractures of the ring of the axis. A classification based on the analysis of 131 cases. J Bone Joint Surg [Br] 1981; 63: 319-327

${ }^{10}$ Fritzwilliams DCL. Inflammatory dislocation of the atlas. Br Med J 1934; 2: 107-109

11 Gallie WE. Fractures and dislocations of the cervical spine. J Surg Am 1939; 46: 495-499

12 Grob D, Jeanneret B, Aebi M et al. Atlantoaxial fusion with transarticular screw fixation. J Bone Joint Surg [Br] 1991; 73: 972-976

${ }^{13}$ Harms J, Melcher RP. Posterior C1-C2 fusion with polyaxial screw and rod fixation. Spine 2001; 26: 2467-2471

14 Jackson $H$. The diagnosis of minimal atlantoaxial subluxation. Br J Radiol 1950; 23: $672-$ 674

${ }^{15}$ Magerl F, Aebi M, Gertzbein SB et al. A comprehensive classification of thoracic and lumbar injuries. Eur Spine J 1994; 3: 184-201

${ }^{16}$ Magerl F, Seemann PS. Stable posterior fusion of the atlas and axis by transarticular screw fixation. In: Kehr P, Weidner A, eds. Cervical spine. Wien, New York: Springer; 1986

17 Wilke HJ, Kettler A, Claes L. Primary stabilizing effect of interbody fusion devices for the cervical spine: an in vitro comparison between three different cages types and bone cement. Eur Spine J 2000; 9: 410-416

Dr. med. Stefan Matschke

Oberarzt, Leiter der Sektion

Wirbelsäulenchirurgie

Dr. med. Martin Adams

Oberarzt

Prof. Dr. med. Andreas Wentzensen Ärztlicher Direktor

Klinik für Unfall- und

Wiederherstellungschirurgie

Berufsgenossenschaftliche

Unfallklinik Ludwigshafen

Unfallchirurgische Klinik an der

Universität Heidelberg

Ludwig-Guttmann-Straße 13

67071 Ludwigshafen

matschke@bgu-ludwigshafen.de 